\title{
Supply Chain Risk Assessment Based on AHP and Fuzzy Comprehensive Assessment Mode: A Case Study of the Chemical Supply Chain
}

\author{
Ying Lu \\ Student Affairs Department, Harbin University of Commerce, Harbin, 150028, \\ China \\ 0451_ly@sina.com
}

\begin{abstract}
Recently, there have been frequent accidents in energy chemical industry, hence the emergency management become normalized. Risk assessment provides basis for policy makers to make decision in the cases of the uncertain information and emergency. The chemical supply chain are influenced by many risk factors. In order to make the assessment of chemical supply chain risk by combining the qualitative method with quantitative method, this paper summarizes the characteristics of the chemical supply chain, applies analytic hierarchy process (AHP) to establish risk assessment system, carries the quantitative analysis on risk factors and their weights, establishes the fuzzy comprehensive evaluation model. Analysis of examples shows that in the complicated system of a variety of risks, risk assessment model that based on the AHP can realize the assessment of the individual risk and the comprehensive risk. The validity and science of the model can be verified through comparing evaluation results with actual results.
\end{abstract}

Keywords: chemical supply chain, Risk assessment, Analytic hierarchy process, the fuzzy comprehensive assessment model

\section{Introduction}

There are a large number of random factors in business operations, and these factors make a potential threat to the supply chain and bring in the vulnerability of supply chain system $^{[1]}$. Supply chain system is a complex system, which is firstly studied from the risk of supply by foreign scholars. Metchell believes that it is caused by different factors, for example, the different educational levels of employees of the member companies, different countries and the features of the markets will impact on inadequate supply (such as the stability of the market structure, changes in market interest rates, etc.. Zsidisin, et $a l$., defines supply risk as "reduced quality of goods and services caused by not timely supply." Based on the general idea of risk, Philip O'Keeffe divides supply chain risk into two types: controllable risks, which include the qualification of the suppliers, the originator products and services; and uncontrollable risks, which include the terrorist behaviors, serious labors stopping work, natural disasters and so on. Current research mainly focuses on the following issues about the supply chain risk: the mechanism of supply chain risk, the identification of supply chain risk, the conduction of supply chain risk, warning of supply chain risk, the assessment of supply chain risk, control of supply chain risk ${ }^{[2,3]}$. In order to reduce the negative effects of the risks, model description of the supply chain risk and theoretical study of its responsive method become the hot topics of research in recent years .

Some specific industries (e.g., the financial industry) have already accumulated a lot of management experience of supply chain risk. but there are fewer studies on the management of chemical supply chain risk, and lacking a complete theoretical system. 
This paper is based on the theory and methods of supply chain management and risk management, learns from management experience in other industries, studies the related issues of the risk assessment of the chemical industry .

In recent years, accidents in energy and chemical industry showed the momentum of frequency. April 20, 2010, the drilling platform of British Petroleum (BP) with the location in the Mexico Gulf exploded, resulting in a large oil leak in the deep sea and billions of economic losses. The ecosystem in that region was seriously damaged during the four years after the oil spill. On the evening of July 27, 2010, flood caused by the heavy rain destroyed the storerooms of two chemical companies in Jilin Province of China, resulting in 7,071 material barrels being washed into the Songhuajiang River, and some barrels contain alkyl trimethyl chloroquine, hexamethyl disilazane and so on. In fact, it is an unavoidable fact that the chemical industry is one of the high-risk industries, for that the serious consequences of frequent accidents in the energy and chemical industry caused wide public concern .

Chemical supply chain possesses the characteristics of long cycle of supply chain cycle, being sensitive to fluctuations in energy, being professional, unstable nature of the product and so on. Time needs arranging reasonably because of the long changeovers period of chemical products and the complex steps. The volatility of energy price needs considering because the changes in raw materials will greatly affect the whole industry chain $^{[4]}$, hence, the downstream companies will store a lot of raw materials to satisfy their strong demands in the rise period, to the opposite, the stocks tend to fall and will increase their market outlook which are the great impacts on the outlook ${ }^{[5]}$. From the perspective of logistics, different products target differently and belong to special logistics; from the perspective of production, it is important to notice various limitations and to be familiar with physical and chemical properties of the products. These characteristics determine that the chemical supply chain requires higher risk management, while the risk of chemical supply chain is characterized by multi-source and uncertainty .

Analytic Hierarchy Process (AHP) is proposed by an American Operations Researcher, Professor TL Saaty. Its basic principle is to decomposite complex problems into composition factors, and to group these factors by relations of domination, thus forming an orderly hierarchical structure. This paper utilizes the basic principles of AHP to analyze factors of influencing the risk of chemical supply chain, to make sure the weights of risk indicators and to make fuzzy assessment on this basis.

\section{Analytic Hierarchy Process and Fuzzy Comprehensive Assessment}

\subsection{Analytic Hierarchy Process}

AHP is an effective method to determine the weights of the coefficients, especially suitable for complex problems that may be difficult to analyze the quantitative indicators. AHP divides various factors of complex problems into orderly layers which connect with each other and make them principled, it gives a quantitative representation on the relative importance of each level based on the fuzzy judgment on the objective reality, and then determines the weights of the relative importance of all the elements by the means of mathematical methods ${ }^{[6]}$. The main steps are as follows :

(1) The factors that involved in the complex problem are divided into several levels to establish a multilevel hierarchy model (target layer, the judge layer, program layer ).

(2) Scale and description. The importance of any two factors in the same level should be judged and quantified when they are compared with each other.

(3) The elements belonging to the same level of hierarchy should be made the pairwise comparison on the criteria of superior elements, and their relative importance are determined by the evaluation scale and the judgment matrix can be constructed. 
(4)The relative importance of each layer elements (weights) is determined by calculating the feature vector of judgment matrix.

(5)Finally, the optimal solution is determined by calculating the integrated importance (weight ) in accordance with the principle of maximum weight.

\subsection{Fuzzy Comprehensive Assessment}

Fuzzy comprehensive assessment is based on fuzzy mathematics ${ }^{[7]}$. It is a comprehensive assessment method by the principle of fuzzy relationship to quantify some ill-defined and unquantitative factors ${ }^{[8]}$. The main steps are as follows :

(1) establish the set of assessment factors.

(2)establish the set of comment.

(3)determine the weight set of assessment index.

(4) implement the expert assessment by the opinion poll.

(5)establish the assessment matrix.

(6) make comprehensive assessment by a mathematical model .

(7)make the normalized processing and obtain the comprehensive and comparable assessment results $[9,10]$.

A large number of complex phenomena and interactions of various factors are involved in the comprehensive assessment on the chemical supply chain risk, furthermore, there are a lot of blur phenomena and fuzzy concepts in this assessment. Therefore, fuzzy assessment matrix can be constructed for quantitative treatment in a comprehensive assessment.

\section{Establish a Hierarchy Model of Risk Assessment}

\subsection{Analysis of Chemical Supply Chain Risk}

The risks in chemical supply chain of chemical companies can be analyzed by methods of social surveys, literature review and expert analysis, and combining with the actual situation of the chemical companies. The risks in the chemical supply chain mainly reflect in the following five aspects:

\section{(1) Risks of Production}

Risks of production contain equipment risks, the capacity risks, the risks of inconsistency of supply and demand. Due to the special characteristics of chemical products, chemical equipments face such risks as thinning by corrosion, cracking by stress and corrosion, high temperature hydrogen corrosion, external damage, brittle fracture. Supply chain is composed of a number of companies, if the production is reduced or stopped because of the capacity of a company and that will inevitably affect the entire supply chain, which is the capacity risk. Chemicals raw materials are often used widely and in high demand, which usually caused the shortage of these raw materials, and the demand is uncertain, inconsistency of supply and demand also likely to bring in production risk.

\section{(2) Risks in Transportation}

According to statistics, more than $95 \%$ of the chemicals need off-site transportation, the distance between the place of origin and the place of sale brings about the long logistics distance and high transportation costs. As to the transportation, most chemicals are transported to the consumer mainly by rail, highway, waterway and exported overseas through foreign trades. As to characteristics of goods, the transportation of chemical industry is in large volume but with less value-added products. A significant number of chemicals have specialized logistics technical requirements and many of them are flammable, explosive and corrosive. The improper means of transportation could lead to 
the expansion of risks, the risks of leakage or explosion may be caused by the behaviors such as falling, dragging, scrolling in the process of handling chemicals; and by the accidents of collisions in the process of transportation.

\section{(3) Risks in Cooperation}

As independent economic entities, supply chain members are not only the long-term partners, but also the competitors. They sometimes deliberately conceal data or provide false data for their own interests, resulting in asymmetry of information and mutual game among the members. In addition to the contracts and agreements, cooperation between enterprises relies more on the mutual trust and other's credibility. Supply chain, as a dynamic enterprise alliance, is a relatively loose relationship. So the original partnership will be destroyed if individual companies quit from the supply chain for some reason, and the disintegration of the supply chain will be resulted from some immoral behaviors of enterprises in the supply chain, such as passive cooperation, disclosure of confidential, breach of contracts.

\section{(4) Risks of Contamination}

Environmental pollution would be caused by operational errors in the production process and some emergencies. Higher security requirements of chemical products for the storage and transportation need specialized equipment of storage and transportation, otherwise the accident may cause pollution.

\section{(5) Environmental Risks}

Environmental risks are primarily originated from changes in laws, regulations and policies; also from the changes in industry environment, earthquakes, fires, floods, snow, hurricanes and other natural disasters. Environmental risks could cause a huge impact on the enterprises in chemical supply chain.

\subsection{Risks Assessment System on Chemical Supply Chain}

Based on the analysis above, assessment system of risks in chemical supply chain established (i.e., hierarchical structure evaluation mode, shown in Figure 1), this model is divided into three levels from the top down, namely the target layer, primary indicators(5) and secondary indicators (15). 


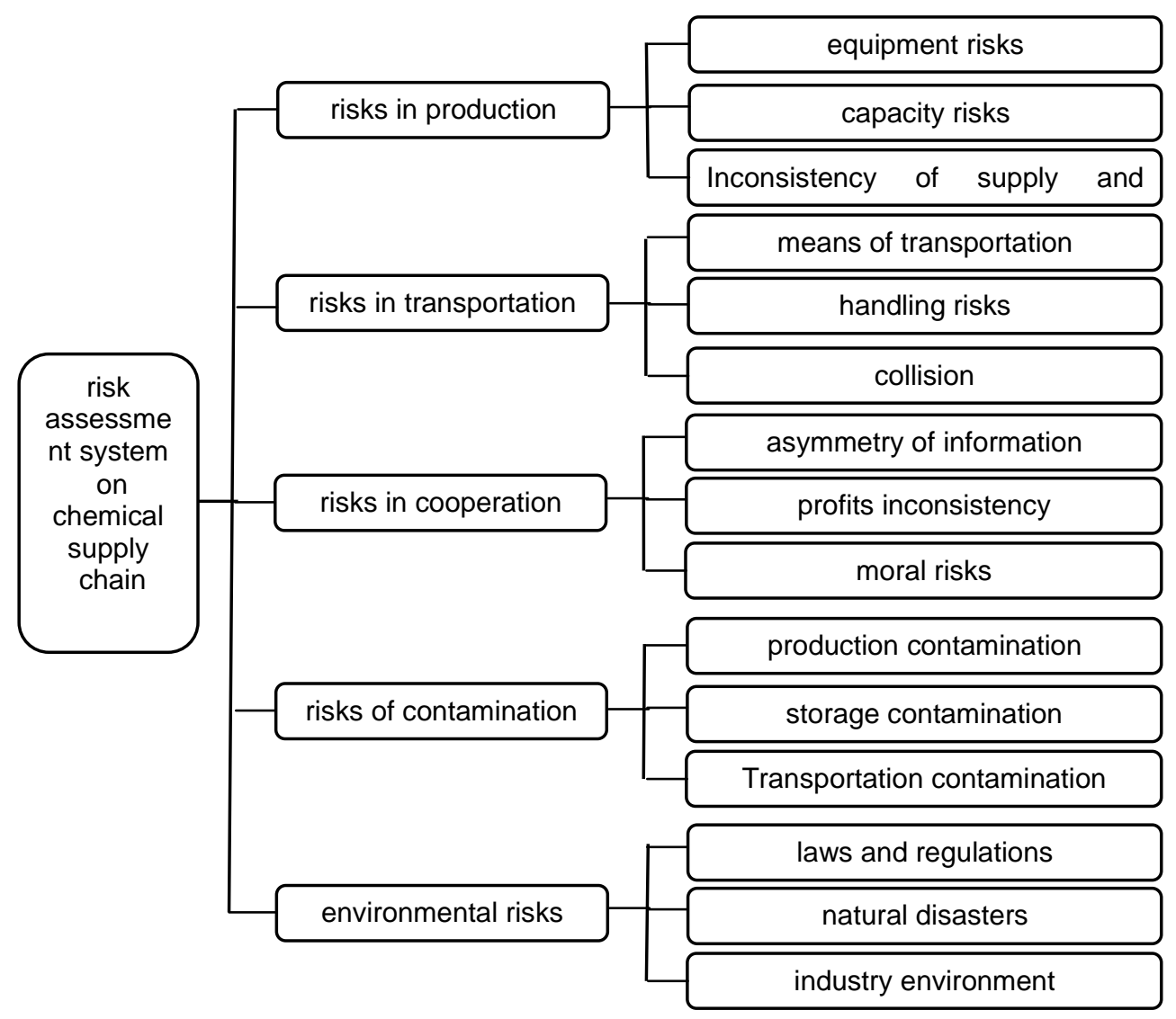

Figure 1. Risk Assessment System on Chemical Supply Chain

\section{Determine the Index Weights of Each Level}

Making the pairwise comparison on factors in the same level and creating comparison judgment matrix ${ }^{[11]}$, concluding the weights of factors in this level to those in superior level by matrix calculation ${ }^{[12]}$.

\subsection{Calculate the Weights of Primary Indicators}

Making the pairwise comparison on five primary indicators respectively, including the risks in production, the risks in transportation, the risks in cooperation, the risk of contamination and environmental risks by the means of experts assessment on the weight and the application of software matlab. We can get the judgment matrix and calculate the weights of the index based on this matrix. The results are shown in Table 1 .

Table 1. Judgment Matrix of First Grade Indexes and their Weights

\begin{tabular}{llllllll}
\hline $\boldsymbol{A}$ & \multicolumn{1}{c}{$\boldsymbol{B 1}$} & $\boldsymbol{B 2}$ & $\boldsymbol{B 3}$ & $\boldsymbol{B 4}$ & $\boldsymbol{B 5}$ & $\boldsymbol{W}$ \\
\hline $\boldsymbol{B 1}$ & 1 & 2 & 3 & 3 & 4 & 0.4048 \\
$\boldsymbol{B 2}$ & $1 / 2$ & 1 & 2 & 2 & 2 & 0.2299 \\
$\boldsymbol{B 3}$ & $1 / 3$ & $1 / 2$ & 1 & 1 & 2 & 0.1389 \\
$\boldsymbol{B 4}$ & $1 / 3$ & $1 / 2$ & 1 & 1 & 2 & 0.1389 \\
\hline
\end{tabular}




\begin{tabular}{lllllll}
\hline B5 & $1 / 4$ & $1 / 2$ & $1 / 2$ & $1 / 2$ & 1 & 0.0875 \\
\hline
\end{tabular}

Consistency test on judgment matrix after calculating the weight, the test is as follows: Largest eigenvalues:

$$
\lambda_{\max }=\frac{1}{\mathrm{n}} \sum_{i=1}^{n} \frac{\left(A \omega_{i}\right)_{i}}{\omega_{i}}=5.0586
$$

Consistency index:

$$
C I=\frac{\lambda_{\max }-n}{n-1}=0.0147
$$

From the table: $R I=1.12$

Consistency ratio:

$$
C R=\frac{C I}{R I}=0.0131
$$

$\mathrm{CR}<0.1$ the consistency is acceptable.

Weight vector $\mathrm{W}=\left(\begin{array}{lllll}0.4048 & 0.2299 & 0.1389 & 0.1389 & 0.0875\end{array}\right)^{\mathrm{T}}$ 。

\subsection{Calculate the Secondary Index Weights}

We can get the eigenvalues and eigenvectors of indicators on the second layer on the same principle and calculation, as shown in Table 2.

Table 2. Characteristic Value and Characteristic Vector of Secondary Indexes

\begin{tabular}{llllll}
\hline $\boldsymbol{K}$ & $\mathbf{1}$ & $\mathbf{2}$ & $\mathbf{3}$ & $\mathbf{4}$ & $\mathbf{5}$ \\
\hline $\boldsymbol{W}_{\boldsymbol{K}}$ & 0.6483 & 0.2970 & 0.0890 & 0.6442 & 0.2297 \\
& 0.2297 & 0.1634 & 0.5876 & 0.2706 & 0.1220 \\
& 0.1220 & 0.5396 & 0.3234 & 0.0852 & 0.6483 \\
$\boldsymbol{A}_{\max }$ & 3.0037 & 3.0092 & 3.0092 & 3.0536 & 3.0037 \\
$\boldsymbol{C l}$ & 0.0018 & 0.0046 & 0.0046 & 0.0268 & 0.0018 \\
$\boldsymbol{C R}$ & 0.0036 & 0.0088 & 0.0088 & 0.0516 & 0.0036 \\
\hline
\end{tabular}

Determine the degree of consistency in the Table : $0.0036<0.1 ; 0.0088<0.1 ; 0.0088$ $<0.1 ; 0.0516<0.1 ; 0.0036<0.1$.

It shows that there is a satisfactory consistency in each matrix.

\section{Fuzzy Assessment}

\subsection{Determine the Assessment Grade}

Suppose there are $\mathrm{N}$ experts making assessment on various indicators of a set of factors, the risks can be divided into five grades of low, comparatively low, medium, comparatively high, high in order to facilitate the assessment.

Comment set $\mathrm{E}=\{\mathrm{e} 1, \mathrm{e} 2, \mathrm{e} 3, \mathrm{e} 4, \mathrm{e} 5\}=\{$ low, comparatively low, medium, comparatively high, high $\}$.

The following is the example analysis of an enterprise. 


\subsection{Construct the Fuzzy Assessment Matrix}

A evaluation group of 20 experts will evaluate the risks of 15 secondary indicators in a chemical supply chain, we can get the evaluation grade RK through statistical arrangement and normalized processing

Table 3. Summary of Risk Assessment

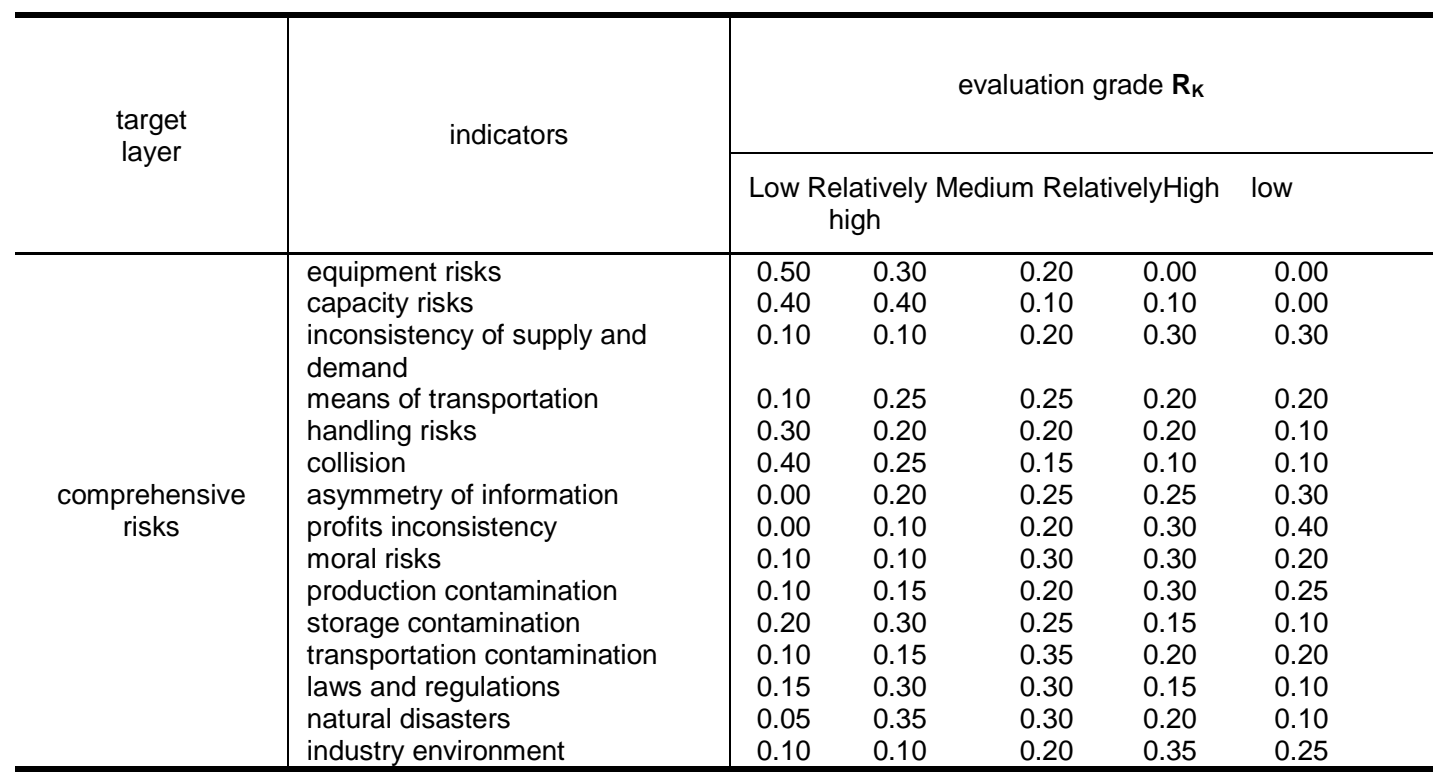

\subsection{Individual Risk Assessment}

Individual risk assessment is an evaluation on risk indicators of primary level. According to the above example, the results of the five primary risk indicators and their corresponding risk levels are as follows:

(1)Risks in Production

$\mathrm{P}_{1}=\mathrm{W}_{1} \cdot \mathrm{R}_{1}=\left(\begin{array}{lllll}0.5 & 0.3 & 0.2 & 0.1220 & 0.1220\end{array}\right)^{\mathrm{T}}$

Maximum membership value: 0.5 , corresponding to "low risk"

(2) risks of transportation

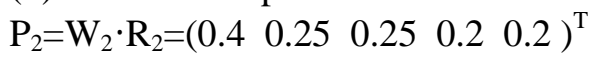

Maximum membership value: 0.4 , corresponding to "low risk"

(3) risks of cooperation

$\mathrm{P}_{3}=\mathrm{W}_{3} \cdot \mathrm{R}_{3}=\left(\begin{array}{lllll}0.1 & 0.1 & 0.3 & 0.3 & 0.4\end{array}\right)^{\mathrm{T}}$

Maximum membership value: 0.4 , corresponding to "high risk "

(4) risks of contamination

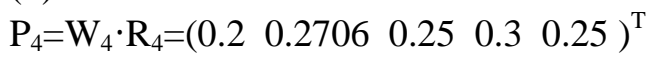

Maximum membership value: 0.3 , corresponding to "comparatively high risk "

(5) environmental risks

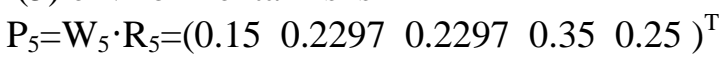

Maximum membership value: 0.35 , corresponds to the " comparatively high risk "

\subsection{Comprehensive Fuzzy Risk Assessment}

The process of comprehensive risk assessment on this chemical supply chain is as follows :

$\mathrm{P}=\mathrm{W} \cdot \mathrm{R}$

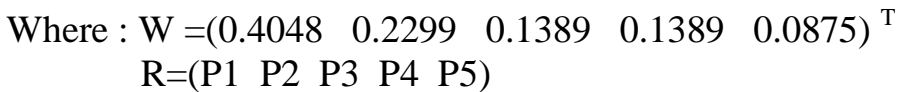




$$
\mathrm{P}=\left(\begin{array}{lllll}
0.2927 & 0.3077 & 0.1389 & 0.2130 & 0.1899
\end{array}\right)^{\mathrm{T}}
$$

Maximum membership value is 0.3077 and corresponds to the "comparatively low risk ", indicating that the risk of this chemical supply chain is comparatively low.

\section{Conclusions}

The results show that in complex systems with the coexistence of a variety of risks, the fuzzy comprehensive evaluation model which is based on AHP can assess the individual risk and comprehensive risks. The validity and scientific value of this model can be proved through the comparison between the assessment results and the actual results.

Frequent accidents in chemical and petrochemical industry require the normalization of the environmental emergency work. We have to make the further study on the relationship among various risks in chemical supply chain, and make the in-depth research on emergency management in the future. We have to further improve the emergency plan and make the experts and technical reserves.

\section{References}

[1]. E. P. Schulz, M. S. Diaz and J. A. Bandoni, "Supply chain optimization of large-scale continuous processes", Computers \& Chemical Engineering, vol. 29, no. 6, (2005), pp. 1305-1316.

[2]. R. Boute, R. V. Dierdonck and A. Vereecke, "Organizing", International Journal of Logistics Research and Applications, vol. 14, no. 5, (2011), pp. 297-315.

[3]. F. You, L. Tao, D. J. Graziano and S. W. Snyder, "Optimal design of sustainable cellulosic biofuel supply chains: Multiobjective optimization coupled with life cycle assessment and input-output analysis", AIChE J, vol. 58, no. 4, (2012), pp. 1157-1180.

[4]. H. Lakkhanawat and M. J. Bagajewicz, "Financial risk management with product pricing in the planning of refinery operations", Industrial \& Engineering Chemistry Research, vol. 47, no. 17, (2008), pp. 66226639.

[5]. A. Pongsakdi, P. Rangsunvigit, K. Siemanond and M. J. Bagajewicz, "Financial risk management in the planning of refinery operations", International Journal of Production Economics, vol. 103, no. 1, (2006), pp. 64-86.

[6]. F. Yang, "Integrated Geographic Information Systems- Based Suitability Evaluation of Urban Land Expansion: A Combination of Analytic Hierarchy Process and Grey Relational Analysis," Environmental Engineering Science, vol. 26, no. 6, (2009), pp. 1025-1032.

[7]. H. V. Enrique, C. Francisco and H. Francisco, et al., "Group decision-making model with incomplete fuzzy preference relations based on additive consistency", IEEE Trans on Systems, Man and Cybernetics, Part B: Cybernetics, vol. 37, no. 1, (2007), pp. 176-189.

[8]. Q. Zhou, W. L. Huang and Y. Zhang, "Identifying critical success factors in emergency management using a fuzzy DEMATEL method", Safety Science, vol. 49, no. 2, (2011), pp. 243 - 252.

[9]. T. L. Saaty and L. T. Tran, "On the invalidity of fuzzifying numerical judgments in the analytic hierarchy process", Mathematical and Computer Modelling, vol. 46, no. 10, (2007), pp. 962 - 975.

[10].T. C. Wang and H. D. Lee, "Developing a fuzzy TOPSIS approach based on subjective weights and objective weights", E3cpert Systems with Applications, vol. 36, no. 5, (2009), pp. 8980 - 8985.

[11].P. Kumar and R. K. Singh, "A fuzzy AHP and TOPSIS methodology to evaluate 3PL in a supply chain", Journal of Modelling in Management, vol. 7, no. 3, (2012), pp. 287-303.

[12].S. Zaim, A. Turkyilmaz, M. F. Acar, U. A. Turki and O. F. Demirel, "Maintenance strategy selection using AHP and ANP algorithms: a case study", Journal of Quality in Maintenance Engineering, vol. 18, no. 1, (2012), pp. 16-2.

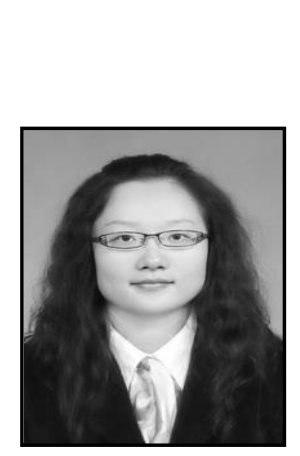

\section{Author}

Ying Lu, Master, work in Student Affairs Department, Harbin University of Commerce, China; associate professor. The major research fields: artificial intelligence, information services; ecommerce and e-government. 